Hiroshi Umemura

Nagoya Math. J.

Vol. 54 (1974), 123-134

\title{
A THEOREM OF MATSUSHIMA
}

\author{
HIROSHI UMEMURA*
}

In [7], Matsushima studied the vector bundles over a complex torus. One of his main theorems is: A vector bundle over a complex torus has a connection if and only if it is homogeneous (Theorem (2.3)). The aim of this paper is to prove the characteristic $p>0$ version of this theorem. However in the characteristic $p>0$ case, for any vector bundle $E$ over a scheme defined over a field $k$ with char. $k=p$, the pull back $F^{*} E$ of $E$ by the Frobenius endomorphism $F$ has a connection. Hence we have to replace the connection by the stratification (cf. (2.1.1)). Our theorem states: Let $A$ be an abelian variety whose $p$-rank is equal to the dimension of $A$. Then a vector bundle over $A$ has a stratification if and only if it is homogeneous (Theorem (2.5)).

The author wishes to express his thanks to T.Oda. The discussion with him was indispensable.

\section{§ 1. Preliminaries}

(1.1) All schemes are of finite type over an algebraically closed field $k$, unless the contrary is stated as in (1.4.1). Let char. $k=p \geq 0$. Let $P$, $X$ be schemes. Let $\pi: P \rightarrow X$ be a morphism. Let $G$ be a group scheme. We denote by $P(X, G, \pi)$ a principal $G$-bundle over $X: G$ operates on $P$ from the right satisfying the following conditions:

(i) The diagram

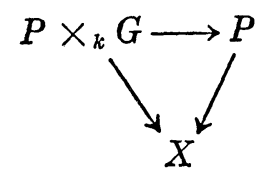

is commutative.

Received October 5, 1973.

* partially supported by the Sakkokai Foundation. 
(ii) The morphism

$$
\begin{array}{r}
G \times{ }_{k} P \longrightarrow P \times{ }_{X} P \\
(g, p) \longmapsto(p \cdot g, p)
\end{array}
$$

is an isomorphism.

(iii) There exists an (fpqc) extension $X^{\prime} \rightarrow X$ such that $P^{\prime}=P \times_{X} X^{\prime}$ is isomorphic to $G \times{ }_{k} X^{\prime}$.

(1.1.1) Let $K$ be a group scheme. Let $H$ be a subgroup scheme. Then the quotient $K \stackrel{\pi}{\longrightarrow} K / H$ exists (S.G.A.D. Exposé $\mathrm{VI}_{A}$ ) and $K(K / H, H, \pi$ ) is a principal $H$-bundle.

We need some results of Grothendieck [4].

(1.2) THEOREM A. An (fpqc) (faithfully flat and quasi-compact) morphism is a strict descent morphism for the category of affine schemes and for the category of quasi-coherent sheaves.

In particular this means two important things:

(1.2.1) Let $S^{\prime}, S$ be schemes. Let $\alpha: S^{\prime} \rightarrow S$ be an (fpqc) morphism. Let $X, Y$ be schemes affine over $S$. Let $X^{\prime}$ (resp. $Y^{\prime}$ ) be the pull-back of $X$ (resp. $Y$ ). Then to define a morphism $X \rightarrow Y$ over $S$, it is sufficient to define a morphism $X^{\prime} \rightarrow Y^{\prime}$ over $S^{\prime}$ commuting with the descent data.

(1.2.2) In the category of affine schemes (or in the category of quasicoherent sheaves) descent data descends through an (fpqc) morphism.

(1.3) Let $P(X, G, \pi)$ be a principal $G$-bundle over $X$ with group $G$. Let $G^{\prime}$ be a group scheme. Let $\varphi: G \rightarrow G^{\prime}$ be a morphism of group schemes.

LEMMA (1.3.1) Assume that $G, G^{\prime}$ are affine over $k$. Then there exists a unique principal $G^{\prime}$-bundle $P^{\prime}\left(X, G^{\prime}, \pi^{\prime}\right)$ such that there exists a morphism $f$ of $P$ to $P^{\prime}$ over $X$ with $f\left(x^{g}\right)=f(x)^{\varphi(g)}, x \in P, g \in G$.

Proof. Let $X^{\prime} \rightarrow X$ be an (fpqc) morphism trivializing $P$. Let $P^{*}$ denote the pull-back of $P$ on $X^{\prime}$. The pull-back $P^{*} \simeq G \times{ }_{k} X^{\prime}$ carries naturally a descent data, so that it induces a descent data on $G^{\prime} \times_{k} X^{\prime}$. We put $P^{* \prime} \simeq G^{\prime} \times{ }_{k} X^{\prime}$. Since $P^{* \prime}$ is affine over $X^{\prime}$, by (1.2.2), $P^{* \prime}$ descends and defines a principal $G^{\prime}$-bundle over $X$. This shows the existence.

We shall show the uniqueness. Let $P_{1}^{\prime}$ and $P_{2}^{\prime}$ be two principal $G^{\prime}$ bundles over $X$ having the property described in the lemma. Let $X^{\prime} \rightarrow$ $X$ be an (fpqc) morphism trivializing $P, P_{1}$ and $P_{2}$. We denote the pull- 
back by ${ }^{*}$. We have $f_{i}^{*}: P^{*} \simeq X^{\prime} \times G \rightarrow X^{\prime} \times G^{\prime}, i=1,2$.

$$
(x, g) \rightarrow\left(x, f_{i}^{*}(x, g)\right)
$$

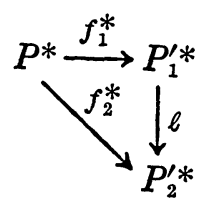

$f_{i}^{*}(x, g)=f_{i}^{*}\left((x, 1)^{g}\right)=f_{i}^{*}(x, 1)^{\varphi(g)}, x \in X^{\prime}, g \in G$. If we put $\ell\left(x, g^{\prime}\right)=$ $\left(x, f_{2}^{*}(x, 1) f_{1}^{*}(x, 1)^{-1} g^{\prime}\right)$ for $\left(x, g^{\prime}\right) \in X^{\prime} \times G^{\prime}$, then the diagram above commutes and gives an isomorphism between $P_{1}^{\prime *}$ and $P_{2}^{\prime *}$. It is easy to check that this isomorphism commutes with the descent data for $P_{1}^{\prime *}$ and for $P_{2}^{\prime *}$. Hence this gives an isomorphism of $P_{1}^{\prime}$ and $P_{2}^{\prime}$ over $X$.

(1.4) Let $X$ be a projective scheme over $k$. Let $P(X, G L(r, k), \pi)$ be a principal $G L(r, k)$-bundle over $X . \quad \widetilde{G}(P)$ is a functor from the category of $k$-schemes to the category of groups defined by the following formula: $\tilde{\mathscr{G}}(p)(T)=\left\{f \in \operatorname{Hom}_{T}\left(P \times{ }_{k} T, P \times_{k} T\right) \mid f\right.$ is an automorphism of $P \times_{k} T$. $f\left(x^{g}\right)=f(x)^{g}$ for any $g \in G L(r, k)$. $\}$ for a scheme $T$ over $k$. Since $X$ is the quotient of $P$ by the action of $G L(r, k), f$ induces the following commutative diagram:

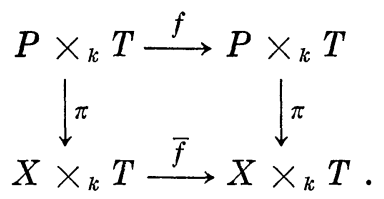

Proposition (1.4.1) The functor $\check{\mathscr{G}}(P)$ is represented by a scheme locally of finite type over $k$.

Proof. $G L(r, k)$ is an open subscheme of the scheme of $r \times r$ matrices $M(r \times r, k)$. $G L(r, k)$ operates on $M(r \times r, k) \simeq A_{k}^{r^{2}}$ from the left and the right as linear automorphisms of the affine space $A_{k}^{r^{2}}$. Since a linear automorphism of $\boldsymbol{A}_{k}^{r^{2}}$ can be prolonged to an automorphism of $\boldsymbol{P}_{k}^{r^{2}}$, the actions from the left and the right of $G L(r, k)$ on $\boldsymbol{A}_{k}^{r^{2}}$ can be extended equivariantly to the actions on $\boldsymbol{P}_{k}^{r^{2}}$. Hence $G L(r, k) \subset \boldsymbol{P}_{k}^{r^{2}}$ is an equivariant completion of $G L(r, k)$ with respect to the both actions. Since a principal $G L(r, k)$-bundle is locally trivial for the Zariski topology, 
we can associate $\boldsymbol{P}_{k}^{r^{2}}$-bundle $P^{\prime}=P \times{ }^{G L(r, k)} \boldsymbol{P}^{r^{2}} \stackrel{\pi^{\prime}}{\longrightarrow} X$ with $P(X, G L(r, k), \pi)$. Then $P$ is an open subscheme of $P^{\prime}$ and $G L(r, k)$ operates on $P^{\prime}$ from the right. From the existence of the Hilbert scheme (See Grothendieck [5]), we deduce that the functor $\operatorname{Aut}_{k} P^{\prime}$ is represented by a group scheme $G$ locally of finite type over $k$ since $P^{\prime}$ is projective over $X$ and since $X$ is projective over $k$ by hypothesis. Now let $Y=P^{\prime}-P$ and we regard $Y$ as a reduced closed subscheme of $P^{\prime}$. Consider the subfunctor $F$ of $\operatorname{Aut}_{k} P^{\prime}$ :

$F=$ Automorphisms of $P^{\prime}$ leaving the closed subscheme $Y$ fixed . Then $F$ is representable. Now we show $\tilde{\mathscr{G}}(P)$ is a subgroup functor of $F$. It is sufficient to show that any element of $\tilde{\mathscr{G}}(P)$ can be extended to an automorphism of $P^{\prime}$. First we assume that $P$ is trivial. In this case, letting $f$ be an element of $\widetilde{\mathscr{G}}(P), f(g)=f\left(I_{r} g\right)=f\left(I_{r}^{g}\right)=f\left(I_{r}\right)^{g}=$ $f\left(I_{r}\right) g$. Hence $f$ is nothing but the multiplication by $f\left(I_{r}\right)$ from the left. Since $P^{\prime}$ is an equivariant completion, the multiplication by $f\left(I_{r}\right)$ can be extended to $P^{\prime}$. In the case $P$ is not trivial, take an open covering $\left\{U_{\alpha}\right\}$ of $X$ so that $\pi^{-1}\left(U_{\alpha}\right)=P_{\alpha}$ is trivial. $\tilde{\mathscr{G}}(P)$ acts on $P_{\alpha}$. By what we have seen above, the action of $\tilde{G}(P)$ on $P_{\alpha}$ can be extended to an automorphism of the restriction of $P^{\prime}$ on $U_{\alpha}$. Since the extension is unique, the extensions over $U_{\alpha}$ and $U_{\beta}$ coincide if $U_{\alpha} \cap U_{\beta} \neq \emptyset$. Hence the operation of $\tilde{G}(P)$ on $P$ can be extended to the operation on $P^{\prime}$. This is what we had to show. $G L(r, k)$ operates on $P^{\prime}$ from the right and leaves $Y$ fixed. Hence $G L(r, k)$ is a closed subgroup scheme of $F$ (See S.G.A.D. Exposé $\mathrm{VI}_{B}$ Cor. 1.4.2.). $\tilde{\mathscr{G}}(P)$ is the centralizer of the closed subgroup scheme $G L(r, k)$ of the group scheme $F$. Hence $\tilde{G}(P)$ is representable.

q.e.d.

(1.4.2) We have the natural homomorphism of group schemes $q: \tilde{G}(P)$ $\rightarrow$ Aut $X$ as we remarked above. The kernel of this homomorphism is the group $\operatorname{Aut}_{X}(P)$ which is connected and affine over $k$ (See $\mathrm{M}$. Maruyama: On a family of algebraic vector bundles, Number Theory, Algebraic Geometry and Commutative Algebra, in honor of Y. Akizuki Kinokuniya, Tokyo, 1973, 95-146). We apply (1.4.1) to an abelian variety $A$.

COROLlaRY (1.4.3) Let $A$ be an abelian variety. Let $P=$ $P(A, G L(r, k), \pi)$. Consider a subgroup functor $\mathscr{G}(P)$ of $\tilde{G}(P)$ whose value is defined for any $k$-scheme $T$ by the following formula: 
$\mathscr{G}(P)(T)=\left\{\left(x_{T}, f\right) \mid x_{T}\right.$ is a T-valued point of $A . \quad f \in \tilde{G}(P)(T)$ such that the diagram

$$
\begin{gathered}
P \times_{k} T \longrightarrow \frac{f}{\pi \times \operatorname{id}_{T} \downarrow} P \times_{k} T \\
A \times_{k} T \underset{\mathrm{id}_{T} \downarrow}{\longrightarrow} A \times_{k} T \\
\text { commutes }\} .
\end{gathered}
$$

Then the group functor $\mathscr{G}(P)$ is represented by a scheme of finite type over $k$.

Proof. The abelian variety $A$ can be regarded in a natural way as a subgroup scheme of $\mathrm{Aut}_{k} A$. Let $i: A \longrightarrow$ Aut $_{k} A$. From the definition $\mathscr{G}(P)$ is isomorphic to the fibre product $\tilde{\mathscr{G}}(P) \times_{\mathrm{Aut}_{6} A} A$. Since $A$ and Aut $_{A} P$ are connected, $\mathscr{G}(P)$ is isomorphic to the fibre product $\tilde{\mathscr{G}}^{0}(P)$ $\times_{\text {Aut }_{6} A} A$ where $\tilde{\mathscr{G}}^{0}(P)$ is the connected component containing the identity of the group scheme $\mathscr{G}(P)$.

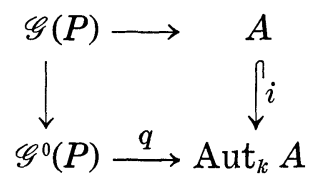

$\tilde{\mathscr{G}}^{0}(P)$ is of finite over $k$. Hence $\mathscr{G}(P)$ is of finite type over $k$. q.e.d.

DEFINITION (1.4.4) Using the notation of (1.4.3),

$$
1 \rightarrow \operatorname{Aut}_{A} P \rightarrow \mathscr{G}(P) \rightarrow A
$$

The quotient group scheme $\mathscr{G}(P) / \mathrm{Aut}_{A} P$ is denoted by $H(P) . \quad H(P)$ is a closed subgroup scheme of $A$ (See S.G.A.D. Exposé $\mathrm{VI}_{A}$ ).

A principal $G L(r, k)$-bundle $P(A, G L(r, k), \pi)$ over an abelian variety $A$ is said to be homogeneous if $H(P)=A$.

If $P$ is homogeneous,

$$
I \rightarrow \operatorname{Aut}_{A} P \rightarrow \mathscr{G}(P) \rightarrow A \rightarrow 0
$$

Remark (1.4.5) The set of all the $k$-valued points of $H(P)$ is $\{k$ valued point $x$ of $A \mid P \simeq T_{x}^{*} P$ where $T_{x}$ is the translation by $\left.x\right\}$.

LEMMA (1.4.6). Let $A, B$ be abelian varieties. Let $\varphi: A \rightarrow B$ be a 
homomorphism. We set $N=\operatorname{Ker} \varphi \stackrel{i}{\longrightarrow} A$ (scheme theoretic kernel, of course). Let $P^{\prime}\left(B, G L(r, k), \pi^{\prime}\right)$ be a principal $G L(r, k)$-bundle over $B$. Let $P(A, G L(r, k), \pi)=\varphi^{*} P^{\prime}\left(B, G L(r, k), \pi^{\prime}\right)$. Then $N$ is a subgroup scheme of $H(P)$.

Proof. Let $f: T \rightarrow N$ be a $T$-valued point of $N$. Then

$$
\begin{aligned}
\left(B \times{ }_{k} T\right) & \times_{T}\left(B \times{ }_{k} T\right) \stackrel{\mu_{B}}{\longrightarrow} B \times_{k} T \\
& \uparrow_{\varphi_{T}} \times \varphi_{T} \\
\left(A \times{ }_{k} T\right) & \times_{T}\left(A \times_{k} T\right) \stackrel{\mu_{A}}{\longrightarrow} A \times_{\varphi_{T}} T \\
& \uparrow_{i_{T}} \times \mathrm{Id} \\
\left(N \times{ }_{k} T\right) & \times_{T}\left(A \times_{k} T\right) \\
& \uparrow_{f_{T} \times \mathrm{Id}}^{\sim} T \\
A \times{ }_{k} T \underset{\Psi}{\stackrel{\sim}{\longrightarrow}} & \times_{T}\left(A \times_{k} T\right),
\end{aligned}
$$

where $\mu_{A}$ (resp. $\mu_{B}$ ) is the law of composition of the abelian scheme $A$ (resp. $B$ ) and $\Psi$ is the natural isomorphism. Hence we get:

$$
\begin{aligned}
f_{T}^{*} P_{T} & =\Psi^{*} \circ\left(f_{T} \times \mathrm{Id}\right)^{*} \circ\left(i_{T} \times \mathrm{Id}\right)^{*} \circ \mu_{A}^{*} P_{T} \\
& =\Psi^{*} \circ\left(f_{T} \times \mathrm{Id}\right)^{*} \circ\left(i_{T} \times \mathrm{Id}\right)^{*} \circ \mu_{A}^{*} \circ \varphi_{T}^{*} P_{T}^{\prime} \\
& \simeq \Psi^{*} \circ\left(f_{T} \times \mathrm{Id}\right)^{*} \circ\left(i_{T} \times \mathrm{Id}\right)^{*} \circ\left(\varphi_{T} \times \varphi_{T}\right)^{*} \circ \mu_{B}^{*} P_{T}^{\prime} \\
& =\varphi_{T}^{*} P_{T}^{\prime} \\
& =P_{T} .
\end{aligned}
$$

This shows we have a section $s$ on $N$ of $\mathscr{G}(P) \rightarrow H(P)$ :

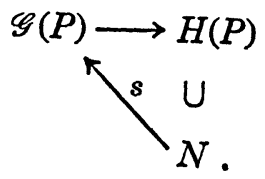

$$
\text { q.e.d. }
$$

Remark (1.4.7) Let $X$ be a scheme. Consider a principal $G L(r, k)$ bundle $P(X, G L(r, k), \pi)$. Let $E$ be the associated vector bundle to $P$. We define the functor $\tilde{\mathscr{G}}(E)$ by the following formula:

$\tilde{\mathscr{G}}(E)(T)=\left\{\left(\varphi, \varphi^{\prime}\right) \mid \varphi\right.$ is a $T$-automorphism of $X \times{ }_{k} T$ and $\varphi^{\prime}$ is an isomorphism of $E_{T}$ to $\left.\varphi^{*} E_{T}\right\}$ for a $k$-scheme $T$. Then it is easy to see that 
$\tilde{\mathscr{G}}(P)=\tilde{\mathscr{G}}(E)$ as group functors. Moreover $\mathrm{Aut}_{X} P=\mathrm{Aut}_{X} E$.

If $X=A$ is an abelian variety, we define a subgroup functor $\mathscr{G}(E)$ of $\tilde{G}(E)$ by the following formula; for any $k$-scheme $T$,

$\mathscr{G}(E)(T)=\left\{\left(\varphi, \varphi^{\prime}\right) \in \tilde{G}(E)(T) \mid \varphi\right.$ is the translation by a $T$-valued point $\left.x_{T}\right\}$.

Then $\mathscr{G}(P)=\mathscr{G}(E)$. Let $H(E)$ be the quotient group scheme of $\mathscr{G}(E)$ by $\mathrm{Aut}_{A} E$. The group schemes $H(P)$ and $H(E)$ are isomorphic. We say that $E$ is homogeneous when $P$ is so (cf. Miyanishi [8], Umemura [10] and Remark (1.4.5)).

\section{\$2. Main theorem}

(2.1) We recall the definition of stratification (Grothendieck [6]).

Definition (2.1.1) Let $X$ be a smooth scheme defined over $k$. Let $E$ be a vector bundle on $X$. For each positive integer $n$, we denote by $\Delta^{1}(n)$ the $n$-th infinitesimal neighbourhood of the diagonal of $X \times_{k} X$. $\Delta^{2}(n)$ denotes the $n$-th infinitesimal neighbourhood of the diagonal of $X \times_{k} X \times_{k} X$. Then we have the usual diagram of projections:

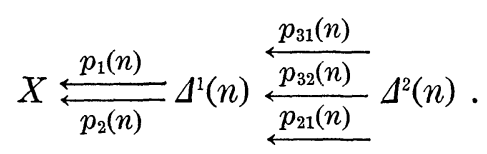

An $n$-connection is an isomorphism

$$
\varphi: P_{1}(n)^{*}(E) \stackrel{\sim}{\longrightarrow} P_{2}(n)^{*} E
$$

satisfying the cocycle condition

$$
P_{31}^{*}(\varphi)=P_{32}^{*}(\varphi) P_{21}^{*}(\varphi) .
$$

A stratification on $E$ is a system of an $n$-connection for each positive integer $n$ so that if $n \leq n^{\prime}$, the $n^{\prime}$-connection induces the given $n$-connection.

We need two facts:

(2.1.2) In the case $k$ is the field of complex numbers $C, E$ has a stratification if and only if $E$ has an integrable connection.

(2.1.3) In the case the characteristic of $k$ is positive, then $E$ has a stratification if and only if $E$ descends through any power of Frobenius 
endomorphism $F^{m}$ of $X$. Sketch of the proof. Consider the fibre product $X^{(m)}$ :

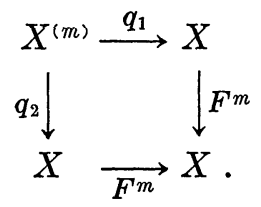

Then $X^{(m)}$ is a closed subscheme of $X \times{ }_{k} X$ defined by the ideal $I^{(m)}$ where $I$ is the ideal defining the diagonal $\Delta^{1}(1)$ and $I^{(m)}$ is the $m$-th Frobenius power, the ideal generated by the $p^{m}$-th power of all the elements of $I$.

Let $E$ be a vector bundle descending through $F^{m}$ for any $m$. Then it induces an isomorphism $q_{1}^{*} E \stackrel{\sim}{\longrightarrow} q_{2}^{*} E$ satisfying the cocycle condition. For any integer $n$, if we take $m$ sufficiently large, we have $\Delta^{1}(n) \subseteq X^{(m)}$ since $\Delta^{1}(n)$ defined by $I^{n}$. The isomorphism $q_{1}^{*} E \stackrel{\sim}{\sim} q_{2}^{*} E$ induces an isomorphism

$$
p_{1}(n)^{*} E=q_{1}^{*} E_{\mid \Delta 1(n)} \stackrel{\sim}{\longrightarrow} q_{2}^{*} E_{\mid \Delta^{1}(n)}=p_{2}(n) * E
$$

satisfying the cocycle condition hence it defines an $n$-connection. Hence $E$ has a stratification.

Conversely, suppose $E$ has a stratification, for any $n$, we have $I^{n} \supset I^{(m)}$ provided $m$ is sufficiently large. Hence we have $X^{(m)} \subseteq \Delta^{1}(n)$. This immersion and the isomorphism $p_{1}(n)^{*} E \stackrel{\sim}{\longrightarrow} p_{2}(n) * E$ give a descent data on $E$. By the descent theory (1.2), $E$ descends through $F^{m}$ for any $m$.

Definition (2.2). Let $G$ be a complex Lie group. Let $B$ be a closed normal subgroup of $G$ such that the quotient $G / B$ is a complex torus $T$. Hence $G$ is a principal $B$-bundle over $T$. Let $\rho: B \rightarrow G L(r, C)$ be a representation of $B$. Let $P=G \times{ }^{B} G L(r, C)$ be the principal $G L(r, C)$-bundle over $T$ associated with this representation. Let $E$ be the vector bundle over $T$ associated with the principal $G L(r, C)$-bundle $P$. We say that the vector bundle $E$ is associated with the Lie group $G$.

The following theorem was proven by Matsushima [7].

THEOREM (Matsushima) (2.3). Let $T$ be a complex torus (not necessarily an abelian variety). Let $E$ be a vector bundle over $T$. 
Then the following are equivalent.

(1) $E$ has a holomorphic connection.

(2) $E$ has an integrable connection.

(3) $E$ is associated with a Lie group.

(4) $E$ is homogeneous.

DEFinition (2.4). Let $G$ be a group scheme. Let $B$ be a normal subgroup scheme such that $B$ is affine and $G / B$ is an abelian scheme $A$. By (1.1.1), $G$ is a principal $B$-bundle over $A$. Let $\rho: B \rightarrow G L(r, k)$ be a representation. Since $B$ and $G L(r, k)$ are affine, we can associate the principal $G L(r, k)$-bundle $P$ over $A$ by Lemma (1.3.1). Let $E$ be the vector bundle associated to the principal $G L(r, k)$-bundle $P$. We say that the vector bundle $E$ is associated to the group scheme $G$.

THEOREM (2.5). Assume that $k$ is of characteristic $p>0$. Let $A$ be an abelian variety defined over $k$. Let $P$ be a principal $G L(r, k)$ bundle over $A$. Let $E$ be the vector bundle associated to $P$. Consider the following conditions.

(1) $E$ has a stratification.

(2) $E$ descends through any power of the Frobenius endomorphism $F^{m}$ of $A$.

(3) $E$ is associated with a group scheme.

(4) $E$ is homogeneous.

Then (3) and (4) are equivalent one another. (1) and (2) are equivalent and they imply (3) and (4).

If the p-rank of $A$ is equal to the dimension of $A$ i.e. if the $p$ linear map $H^{1}\left(A, O_{A}\right) \rightarrow H^{1}\left(A, O_{A}\right)$ induced by the Frobenius endomorphism of $A$ is bijective, then all the conditions are equivalent.

Proof. By (1.2), (1) and (2) are equivalent. We shall show the equivalence of (3) and (4). Assume that $E$ is associated to a group scheme $G$. We use the notation of the Definition (2.4). Let $x$ be a point of $G$. Then the following diagram is commutative:

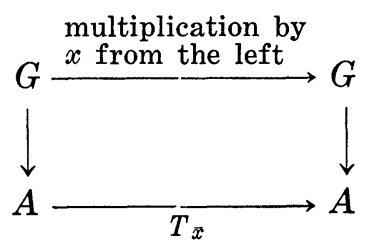


This shows that $G$ is homogeneous. Hence the associated vector bundle to $G$ is also homogeneous.

Now suppose that $P$ is homogeneous. Then we have the exact sequence

$$
1 \rightarrow \operatorname{Aut}_{A} P \rightarrow \mathscr{G}(P) \rightarrow A \rightarrow 0 .
$$

We shall show that $P$ is associated to the group scheme $\mathscr{G}(P)$. Let $r$ be the rank of $E$. We define a representation $\tilde{\alpha}:$ Aut $_{A} P \rightarrow G L(r, k)$ as follows :

Let $x_{0}$ be a $k$-valued point of $P$ lying over 0 in $A$. Let $T$ be a scheme. Consider the map

$$
\begin{array}{cr}
\operatorname{Aut}_{A} P(T) \subset \operatorname{Hom}_{k}\left(P \times_{k} T, P \times_{k} T\right) & \longrightarrow P(T) \\
g & \longrightarrow g\left(x_{0_{T}}\right)
\end{array}
$$

where $x_{0_{T}}: k \times_{k} T \stackrel{x_{0} \times \text { id }}{\longrightarrow} P \times_{k} T$. Then there exists the unique element $\tilde{\alpha}(g)$ of $G L(r, k)(T)$ such that $g\left(x_{0_{T}}\right)=x_{0_{T}}^{\tilde{\alpha}(g)}$. We put

$$
\begin{aligned}
\tilde{\alpha}(T): \operatorname{Aut}_{A} P(T) & \longrightarrow G L(r, k)(T) . \\
g & \longmapsto \tilde{\alpha}(g)
\end{aligned}
$$

Since $\tilde{\alpha}$ is functorial we get a representation $\tilde{\alpha}: \mathrm{Aut}_{A} P \rightarrow G L(r, k)$. We define a morphism of fibre bundles

$$
\begin{aligned}
\alpha: \mathscr{G}(P) & \longrightarrow P . \\
g & \longmapsto g x_{0}
\end{aligned}
$$

Since this is functorial and commutes with the representation $\tilde{\alpha}:$ Aut $_{A} P \rightarrow$ $G L(r, k)$, we get a homomorphism of fibre bundles over $A$. By Lemma (1.3.1), $P$ is the principal $G L(r, k)$-bundle associated to the group scheme $\mathscr{G}(P)$.

We prove (2) $\Rightarrow$ (4). Suppose that $E$ descends through any power of the Frobenius endomorphism $F^{m}$ of $A$. We denote by $N_{m}$ the kernel of $F^{m}$. Then by Lemma (1.4.6), $N_{m} \subset H(E) \subset A$. Hence the formal groups $\hat{H}(E)$ and $\hat{A}$ are isomorphic. This implies $H(E) \simeq A$. Hence $E$ is homogeneous.

Assume that the $p$-rank of $A$ is equal to the dimension of $A$. We shall show (4) $\Rightarrow(2)$. Suppose that $E$ is homogeneous. If $E$ is decomposable, say $E=E_{1} \oplus E_{2} \oplus \cdots \oplus E_{s}$ such that $E_{i}$ is indecomposable for 
$1 \leq i \leq s$, then $E_{i}$ is homogeneous for $1 \leq i \leq s$. In fact if $E_{1}$ were not homogeneous, we would have a closed point $x$ of $A$ such that $T_{x}^{*} E_{1}$ is isomorphic to none of $E_{i}, 1 \leq i \leq s$ since $\left\{x \in A \mid T_{x}^{*} E_{1} \simeq E_{i}\right.$ for some $\left.i\right\}$ would be a closed subset of dimension strictly less than the dimension of $A$ (cf. (1.4.5)). Then we would have $E_{1} \oplus E_{2} \oplus \cdots \oplus E_{s} \simeq E \simeq T_{x}^{*} E$ $\simeq T_{x}^{*} E_{1} \oplus T_{x}^{*} E_{2} \oplus \cdots \oplus T_{x}^{*} E_{s}$. This is a contradiction to the Krull-Schmidt theorem (Atiyah [1]). Hence we may assume $E$ to be indecomposable. Then by Miyanishi [8], $E$ is isomorphic to $F_{r} \otimes L$ where $L$ is a line bundle algebraically equivalent to 0 and $F_{r}$ is a succesive extension of $O_{A}$ by $O_{A}$ :

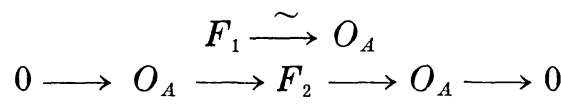

$$
\begin{aligned}
& 0 \longrightarrow F_{2} \longrightarrow F_{3} \longrightarrow O_{A} \longrightarrow 0 \\
& 0 \longrightarrow F_{r-1} \longrightarrow F_{r} \longrightarrow O_{A} \longrightarrow 0 \text {. }
\end{aligned}
$$

Since $L$ descends through any power of the Frobenius endomorphism $F^{m}$ of $A$, it is sufficient to show the

LEMMA (2.6). If the p-linear map $H^{1}\left(A, O_{A}\right) \rightarrow H^{1}\left(A, O_{A}\right)$ induced by $F$ is bijective, then $F_{r}$ descends to $F_{r}^{(m)}, r \geq 1$, through any power of the Frobenius endomorphism $F^{m}$ of $A$ and the $p^{m}$-linear map $H^{i}\left(A, F_{r}^{(m)}\right)$ $\rightarrow H^{i}\left(A, F_{r}\right)$ induced by $F^{m}$ is bijective for any $m, i \geq 0$.

Proof. Induction on $r$. If $r=1$, then $F_{1} \simeq O_{A}$ descends and by hypothesis the map $H^{1}\left(A, O_{A}\right) \rightarrow H^{1}\left(A, O_{A}\right)$ is bijective. Since $H \cdot\left(A, O_{A}\right)=$ $\Lambda \cdot H^{1}\left(A, O_{A}\right)$, we conclude that the map $H^{i}\left(A, O_{A}\right) \rightarrow H^{i}\left(A, O_{A}\right)$ is bijective for any $i \geq 0$. Suppose that the assertion is proven for $r$. We have exact sequence

$$
0 \longrightarrow F_{r-1} \stackrel{\alpha}{\longrightarrow} F_{r} \stackrel{\beta}{\longrightarrow} O_{A} \longrightarrow 0 \text {. }
$$

Since the extension is determined by an element of $H^{1}\left(A, F_{r-1}\right), F_{r-1}$ descends to $F_{r-1}^{(m)}$ and since $F^{m}$ induces a bijective morphism $H^{1}\left(A, F_{r-1}^{(m)}\right)$ $\rightarrow H^{1}\left(A, F_{r-1}\right)$ by induction hypothesis, $F_{r}$ descends to an extension of $O_{A}$ by $F_{r-1}^{(m)}$,

$$
0 \longrightarrow F_{r-1}^{(m)} \stackrel{\alpha^{(m)}}{\longrightarrow} F_{r}^{(m)} \stackrel{\beta^{(m)}}{\longrightarrow} O_{A} \longrightarrow 0
$$


By $\left(^{*}\right),(* *)$ and induction hypothesis we have

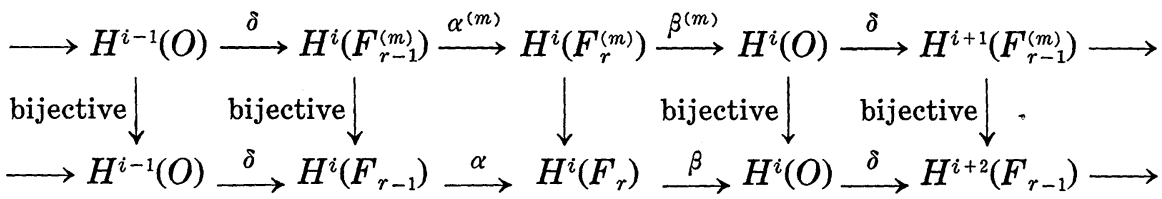

Then by the five lemma, we conclude that $F^{m}$ induces a bijective $p^{m}-$ linear map $H^{i}\left(A, F_{j}^{(m)}\right) \rightarrow H^{i}\left(A, F_{r}^{(m)}\right)$ for any $i$. This is what we had to show.

Remark (2.7). If the $p$-rank of $A$ is not equal to the dimension of $A$, the conditions (2) and (4) in the Theorem (2.5) are not always equivalent. For example, let $A$ be an elliptic curve whose $p$-rank is 0 . Let $F_{2}$ be the unique extension of $O_{A}$ by $O_{A}$ with $H^{0}\left(A, F_{2}\right) \neq 0$ (cf. Atiyah [2]). Then $F_{2}$ is homogeneous. But $F_{2}$ does not descent through the Frobenius endomorphism $F$ of $A$.

\section{REFERFNCES}

[1] Atiyah, M. F., On the Krull-Schmidt theorem with application to sheaves, Bull. Soc. Math. France, 84 (1956), 307-317.

[2] - Vector bundles over an elliptic curve, Proc. Lond. Math. Soc. (3), 7 (1957), 414-452.

[ 3 ] Demazure, M. et Grothendieck, A., Schemas en Groupes (S. G. A. D.), I, II, III, Vol. 151, 152, 153, Lecture Notes in Math., Springer.

[4] Grothendieck, A., Technique de construction et théorèmes d'existence en géométrie algébrique I. Généralités. Descente par morphismes fidèlement plats, Séminaire Bourbaki, t. $12(1959 / 60), \mathrm{n}^{\circ} 90$.

[5] —- Technique de construction et théorèmes d'existence en géométrie algébrique IV. Les schémas de Hilbert, Séminaire Bourbaki, t. $13(1960 / 61), n^{\circ} 221$.

[6] _ Crystals and the De Rham cohomology of schemes, Dix exposés sur la cohomologie des schemas, 1968 North-Holland Pub. company.

[ 7 ] Matsushima, Y., Fibrés holomorphes sur un tore complexe, Nagoya Math. J., vol. 14 (1959), 1-24.

[ 8 ] Miyanishi, M., Some remarks on algebraic homogeneous vector bundles, Number Theory, Algebraic Geometry and Commutative Algebra, in honor of Y. Akizuki, Kinokuniya, Tokyo (1973), 71-93.

[9] Morimoto, A., Sur le groupe d'automorphismes d'un espace fibré principal analytique complexe, Nogaya Math. J., vol. 13 (1958), 157-168.

[10] Umemura, H., Some results in the theory of vector bundles, Nagoya Math. J., vol. 52 (1973), 97-128.

Nagoya University 